\title{
Basolateral amygdala projections to ventral hippocampus modulate the consolidation of footshock, but not contextual, learning in rats
}

\author{
Mary L. Huff, ${ }^{1}$ Eric B. Emmons, ${ }^{2}$ Nandakumar S. Narayanan, ${ }^{2,3}$ and Ryan T. LaLumiere ${ }^{1,2}$ \\ ${ }^{1}$ Department of Psychological and Brain Sciences; ${ }^{2}$ Interdisciplinary Graduate Program in Neuroscience; ${ }^{3}$ Department of Neurology, \\ Carver College of Medicine, University of lowa, lowa City, lowa 52242, USA
}

\begin{abstract}
The basolateral amygdala (BLA) modulates memory consolidation for a variety of types of learning, whereas other brain regions play more selective roles in specific kinds of learning suggesting a role for differential consolidation via distinct BLA pathways. The ventral hippocampus (VH), an efferent target of the BLA, has been suggested to selectively process emotionrelated learning, yet whether the BLA $\rightarrow$ VH pathway modulates memory consolidation, and does so in a learning-specific manner, is unknown. To address this issue, the BLA of male Sprague-Dawley rats was bilaterally transduced to express either ChR2(E123A) or eArchT3.0. Fiber optic probes were implanted in the VH to provide illumination of BLA axons. Rats then underwent a modified contextual fear conditioning task permitting separation of context and footshock learning. On day 1 , rats received $3 \mathrm{~min}$ of pre-exposure to the apparatus. On day 2, rats were placed into the apparatus, received an immediate footshock, and quickly removed. Retention was tested on day 4. Optical stimulation of the BLA $\rightarrow$ VH pathway following footshock, but not context, training using trains of $40-\mathrm{Hz}$ light pulses enhanced retention. Continuous optical inhibition of this pathway for 15 min starting 25 min after footshock training impaired retention. These findings indicate that BLA $\rightarrow$ VH projections influence the consolidation for footshock, but not context, learning of a modified CFC task and provide direct evidence that BLA projections to other brain regions modulate memory consolidation selectively depending on the kind of learning involved.
\end{abstract}

Learning and memory involves complex interactions among multiple brain regions that play discrete roles in the consolidation of specific kinds of memories. However, work strongly indicates that the basolateral amygdala (BLA) modulates the consolidation of a wide array of memories. It has been hypothesized that the BLA plays this more general role, at least in part, through interactions with distinct regions (McGaugh 2002, 2004; McIntyre et al. 2012). BLA connections with forebrain regions such as the hippocampus and striatum that are selectively involved in the consolidation of certain kinds of memories (McDonald 1991; Pitkänen et al. 2000; McGaugh 2002; Malin and McGaugh 2006; Paz et al. 2006; Chavez et al. 2013) suggest that efferent pathways from the BLA may be selectively involved in modulating consolidation for specific kinds of information.

Indeed, much evidence suggests that the BLA interacts with the hippocampus during memory consolidation (Packard et al. 1994; Roozendaal et al. 1999; Malin and McGaugh 2006) and that the BLA directly or indirectly influences activity and plasticity in different parts of the hippocampal formation (Ikegaya et al. 1995; Frey et al. 2001; McIntyre et al. 2005; McReynolds et al. 2014; Lovitz and Thompson 2015). These findings suggest pathway mechanisms by which the BLA influences the consolidation of memories regulated by the hippocampal formation. However, the hippocampus comprises different subregions, including dorsal and ventral divisions, which have been suggested to process different kinds of information (Moser and Moser 1998; Bannerman et al. 2004; Fanselow and Dong 2010). In rodents, the dorsal hippocampus (DH) has been strongly implicated in spatial memories (Kim and Fanselow 1992; Moser et al. 1995;

\section{Corresponding author: mary-I-huff@uiowa.edu}

Article is online at http://www.learnmem.org/cgi/doi/10.1101/lm.039909.115.
Barrientos et al. 2002), whereas investigations into the role of the ventral hippocampus (VH) in spatial processing remain less clear. Some studies propose VH necessity for tasks involving spatial memory (Ferbinteanu and McDonald 2001; Rudy and Matus-Amat 2005), whereas others have discounted the VH as a site for spatial processing (Moser et al. 1995; Pothuizen et al. 2004). In contrast, the VH has been more clearly implicated in processing emotion and anxiety (Henke 1990; Kjelstrup et al. 2002; Bannerman et al. 2004; Maren and Holt 2004). Although much prior work has focused on the interactions between the BLA and DH during memory consolidation, the BLA maintains a direct projection to the $\mathrm{VH}$, but not the DH (Pikkarainen et al. 1999), and recent work supports the functionality of this projection (Felix-Ortiz and Tye 2014). Yet, to our knowledge, no prior study has examined the interaction between the BLA and VH during memory consolidation, and, moreover, it is unknown whether BLA projections to the $\mathrm{VH}$ are selectively involved in the consolidation of specific types of information, but not others.

Contextual fear conditioning (CFC) involves learning regarding both contextual and emotional (footshock) information, suggesting the existence of distinct regions in the brain involved in dissociable components of this learning. Substantial evidence from CFC studies indicates that a brief footshock with minimal time spent in a context produces poor retention of the training, an effect reversed by exposure to the

(C) 2016 Huff et al. This article is distributed exclusively by Cold Spring Harbor Laboratory Press for the first 12 months after the full-issue publication date (see http://learnmem.cshlp.org/site/misc/terms.xhtml). After 12 months, it is available under a Creative Commons License (Attribution-NonCommercial 4.0 International), as described at http://creativecommons.org/licenses/by$\mathrm{nc} / 4.0 /$. 
context before the footshock (Fanselow 1986, 1990; Kiernan and Westbrook 1993; Landeira-Fernandez et al. 2006). Thus, the separate mnemonic components of the task can be investigated through presentation of the context and footshock on separate days. Prior work using this modified CFC paradigm has dissociated roles for the $\mathrm{DH}$ and anterior cingulate cortex. The $\mathrm{DH}$, but not anterior cingulate, is involved in the consolidation of the context learning, whereas the anterior cingulate, but not the $\mathrm{DH}$ is involved in the consolidation of the footshock learning (Malin and McGaugh 2006), establishing the utility of this paradigm in investigating the neurobiology underlying the consolidation of two different kinds of information. Although previous findings indicate a role for the $\mathrm{VH}$ in contextual fear conditioning (Bast et al. 2001; Gilmartin et al. 2012; Zhu et al. 2014), it is unknown whether BLA inputs to the VH influence memory consolidation for CFC and whether they do so in a manner related to the type of information being consolidated. Therefore, the present study used the modified CFC task to separate context and footshock learning (Liang 1999; Malin and McGaugh 2006) and provided optogenetic stimulation or inhibition of BLA axons in the VH immediately after training for either the context or footshock components of the task. It was hypothesized that the BLA projections to the $\mathrm{VH}$ would be involved in the consolidation for the footshock learning, but not the context learning.

\section{Results}

To examine the consolidation for context vs. footshock learning, we used the modified CFC training paradigm, as described in detail in Materials and Methods. Figure 1A depicts the inhibitory avoidance chamber used for the behavioral task, and Figure 1B shows a general timeline of the behavioral training and testing. In brief, rats were placed into the inhibitory avoidance chamber on day 1 and given $3 \mathrm{~min}$ to explore the entire chamber (context pre-exposure). On day 2 , rats were placed into the darkened shock compartment, with no access to the lit safe compartment, and given an immediate footshock (level adjusted for each experiment to prevent ceiling and floor effects). On day 4, rats were placed into the lit "safe" chamber with access to the entire chamber, and their latencies to cross into the darkened chamber were measured and used as the index of retention, with higher latencies indicating better retention.

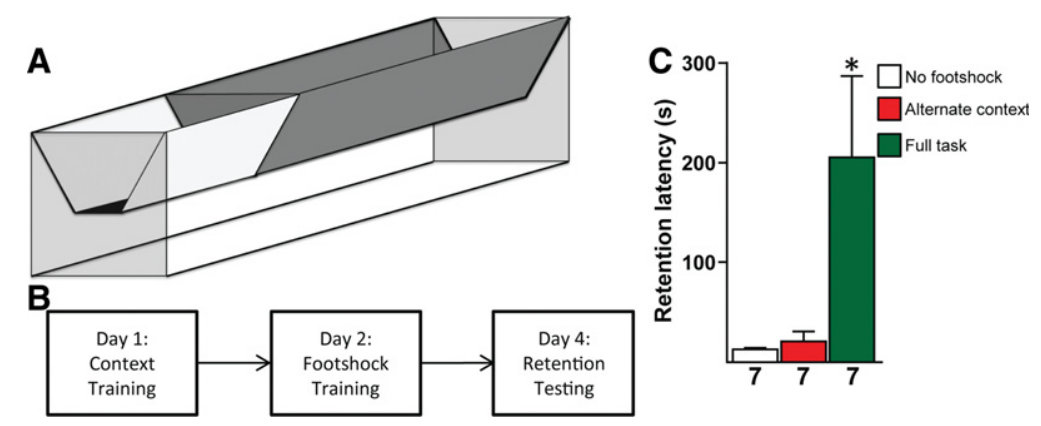

Figure 1. (A) Diagram of inhibitory avoidance apparatus used in the modified CFC training. (B) Schematic diagram of the timeline for behavioral training and testing. (C) Naïve rats (i.e., rats that did not undergo surgeries) underwent the modified CFC task. Rats received either (1) context training on day 1 but no footshock on day 2; (2) alternate-context training on day 1 and footshock training on day 2; or (3) context training on day 1 and footshock training on day 2 (i.e., the full CFC training). The figure shows the mean retention latencies, in seconds $( \pm S E M)$, as well as the number of rats in each group under the respective bar. Those rats that received context and footshock training in the same apparatus had significantly higher retention latencies than the other two groups $\left(^{*}\right) P<0.05$, which did not differ from each other.
To confirm prior work indicating the necessity of both context training (pre-exposure to the entire apparatus) and footshock training (immediate shocks paired with one portion of the apparatus) for retention of the CFC task, naïve rats (i.e., rats that did not undergo surgeries of any kind) either received context training on day 1 with no footshock on day 2 , alternate-context training on day 1 with a footshock on day 2 , or both the context plete CFC training). Figure $1 \mathrm{C}$ shows the day 4 retention latencies, respectively, for all three groups. A one-way ANOVA revealed a significant effect $\left(F_{(2,18)}=5.304, P<0.05\right)$ with post hoc analyses indicating significantly higher latencies for those rats receiving the complete CFC training (both context and footshock training) compared with those of rats receiving either alternate-context training on day 1 or no footshock on day 2 . The retention latencies for the alternate context and no-footshock groups did not differ from each other.

To examine the role of the BLA projection to the VH during memory consolidation, the BLA was transduced with either the depolarizing opsin ChR2(E123A) or the hyperpolarizing opsin eArchT3.0 with optic probes implanted in the VH enabling illumination of the BLA axons, as described in Materials and Methods. all experiments, rats received optical manipulations after either the context or footshock training, either immediately or at some delay following the training (details in Materials and Methods). In brief, upon being removed from the training apparatus (or following the delay, during which rats remained in their home cage), bilaterally implanted fiber optic probes were connected to optical fibers through which light of the appropriate wavelength was delivered. The rat was then placed into an open-top box with opaque

\section{Optical activation of BLA axons in the VH enhances retention of footshock learning for a modified CFC task}

To determine whether post-footshock stimulation of the BLA $\rightarrow$ pathway enhances consolidation for CFC, rats underwent the modified CFC training and received post-training optical stimulation of ChR2(E123A)-transduced BLA terminals in the day 2 , as outlined in Figure 2A. The BLA-VH pathway was stimulated using trains of light pulses (2-sec trains, given every $10 \mathrm{sec}$ ) at either 20,40 , or $80 \mathrm{~Hz}$ (5-msec pulse width, $473 \mathrm{~nm}$ light) over $15 \mathrm{~min}$. Figure 2B shows the retention latencies for rats that underwent the full modified CFC training and received optical stimulation of the BLA axons in the VH immediately following footshock training on day 2. For this experiment, the sham-control group expressed either ChR2(E123A) or eYFP-alone and received no illumination, and these groups were combined for analysis as their latencies did not significantly differ from one another $\left(t_{(12)}=\right.$ $0.057, P>0.05$; mean \pm SEM for each: $\mathrm{ChR} 2(\mathrm{E} 123 \mathrm{~A})=37.50 \pm 10.30 ; \quad$ eYFP $=$ $36.5 \pm 9.78)$. A one-way ANOVA revealed a significant difference between groups $\left(F_{(4,38)}=7.057, \quad P<0.001\right)$. Rats that received trains of $40 \mathrm{~Hz}$ pulses had significantly increased retention latencies compared with sham controls $(P<$ $0.01)$, the $20-\mathrm{Hz}$ group $(P<0.01)$, and eYFP controls $(P<0.01)$. No other groups were significantly different from each other. To determine whether the 

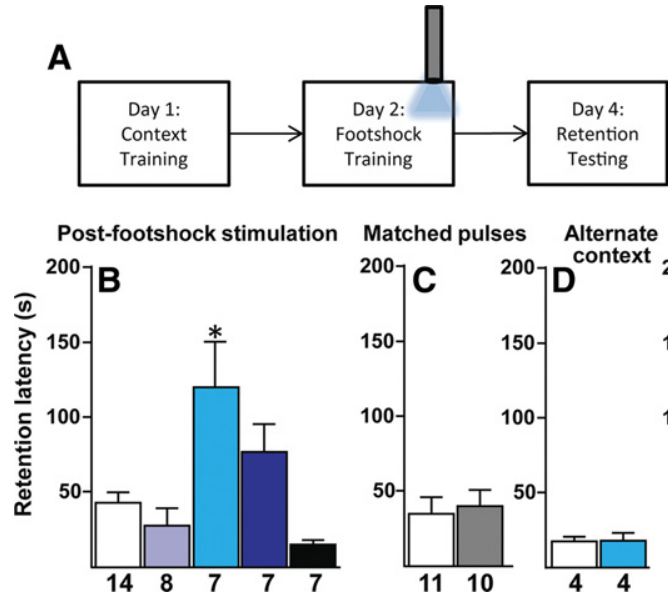

No footshock

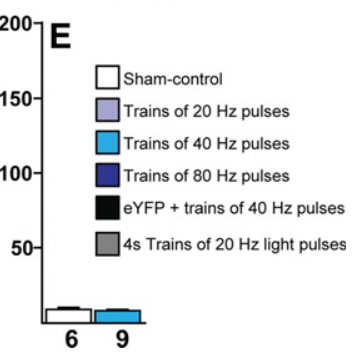

Figure 2. Retention effects of optical stimulation of ChR2(E123A)-transduced BLA axons in the VH immediately after footshock (day 2) training. $(B-E)$ show the mean retention latencies, in seconds $( \pm \mathrm{SEM})$, as well as the number of rats in each group under the respective bar. $(A)$ Schematic diagram of the timeline for experimental training as well as optical stimulation following footshock training. (B) Increased retention latencies of rats given post-footshock training optical stimulation of BLA axons in the VH via activation of ChR2(E123A). Retention was significantly increased in rats that received trains of $40 \mathrm{~Hz}$ light pulses following training. ( $\left.{ }^{*}\right) P<0.05$ compared with sham-control, 20 $\mathrm{Hz}$, and eYFP-control groups. (C) No effect on retention when identical total number of light pulses was given to rats after footshock training. Rats received $4-\mathrm{sec}$ trains of $20-\mathrm{Hz}$ light pulses, thus matching the total number of light pulses given to rats in panel $B$ that received 2-sec trains of $40-\mathrm{Hz}$ pulses. $(D, E)$ No effect of optical stimulation of the BLA $\rightarrow \mathrm{VH}$ pathway following day 2 training on retention if either component of the task (context pre-exposure or footshock delivery, respectively) was altered/omitted.

effect observed with the trains of $40 \mathrm{~Hz}$ stimulation was due to the number of light pulses received (rather than the frequency), a separate experiment (matched pulses) was conducted in which ChR2(E123A)-transduced rats received $20 \mathrm{~Hz}$ stimulation in 4-sec trains following footshock training and thus received an equivalent number of light pulses. The latencies of these rats were not significantly different from the latencies of their shamcontrol rats, as shown in Figure 2C $\left(t_{(19)}=0.34, P>0.05\right)$.

To further demonstrate the necessity of both components of the task, a separate group of rats underwent context training in an alternate context (an operant chamber) on day 1 and then received optical stimulation using trains of $40-\mathrm{Hz}$ light pulses following standard footshock training on day 2 . Retention latencies for the stimulation group, as shown in Figure 2D, were not significantly different from those of the sham-control group $\left(t_{(6)}=0.082, P>0.05\right)$. In addition, to ensure that the optical stimulation of the BLA $\rightarrow \mathrm{VH}$ pathway alone did not induce aversion for the shock chamber, a control experiment was conducted in which rats received normal context training but, on day 2, were simply placed into the shock compartment with no footshock administered. They were then given optical stimulation identical to that found to be effective at enhancing retention in Figure 2B. Retention latencies for those rats, shown in Figure 2E, again did not differ between the sham-control and stimulation groups $\left(t_{(13)}=0.64, P>0.05\right)$.
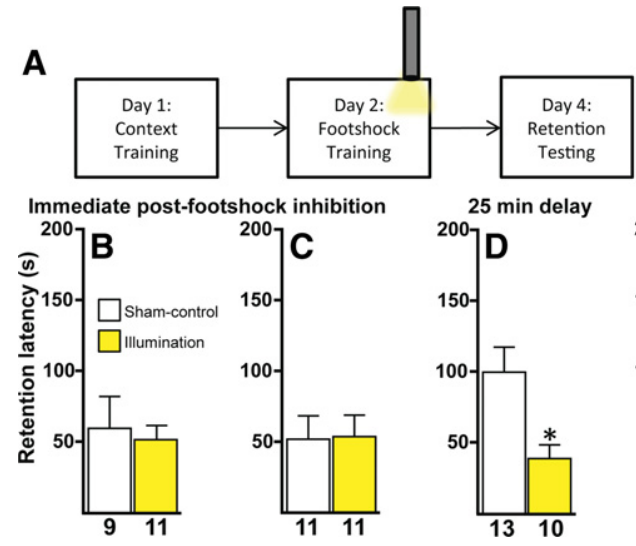

Optical inhibition of BLA axons in the VH impairs retention of footshock learning for a modified CFC task when given at a 25 -min delay

To determine whether post-footshock inhibition of the BLA $\rightarrow \mathrm{VH}$ pathway impairs consolidation of a CFC task, rats underwent $\mathrm{CFC}$ training and received post-training optical inhibition of eArchT3.0-transduced BLA terminals in the $\mathrm{VH}$ following footshock training on day 2 as outlined in Figure 3A. Optical inhibition was obtained using continuous 561-nm light for $15 \mathrm{~min}$. The latencies of rats that received $15 \mathrm{~min}$ of immediate post-footshock training inhibition are shown in Figure 3B, but no significant difference was observed (single footshock: $\left.t_{(18)}=0.34, P>0.05\right)$. Due to the low control group latencies and the potential for a "floor" effect, we repeated the experiment using two footshocks. Latencies for those rats are shown in Figure 3C, and again no significant difference was observed $\left(t_{(20)}=0.076\right.$; $P>0.05$ ).

Regardless of the footshock parameters delivered on day 2 (a single 2-sec, 1 -mA footshock or two $1 \mathrm{sec}, 1 \mathrm{~mA}$ footshocks), the control groups' latencies were very low, potentially creating floor effects that prevented observation of any memory impairment. Therefore, we considered that having a small delay between the footshock and the optical manipulations might lead to higher control latencies, as we have previously observed that immediate post-training optical manipulations produce depressed control baselines (Huff et al. 2013). Moreover, prior work suggests that BLA activity $\sim 30$ min following a learning
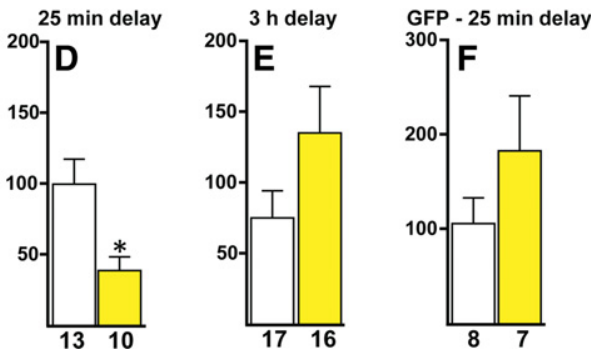

Figure 3. Retention effects of optical inhibition of eArchT3.0-transduced BLA axons in the VH after footshock (day 2) training. ( $B-F$ ) show the mean retention latencies, in seconds ( \pm SEM), as well as the number of rats in each group under the respective bar. (A) Schematic diagram of the timeline for experimental training as well as optical inhibition following footshock training. $(B, C)$ No effect on retention was observed when optical inhibition was provided immediately after footshock training regardless of footshock strength and duration (B: A single $1 \mathrm{~mA}, 2$-sec footshock; C: Two $1 \mathrm{~mA}, 1$-sec footshocks). (D) Impaired retention of rats given post-footshock optical inhibition of BLA axons in the VH at a 25-min delay. $\left(^{*}\right) P<0.05$, compared with sham control. $(E)$ No effect on retention of 15 min of continuous optical inhibition of the BLA $\rightarrow$ VH pathway given $3 \mathrm{~h}$ after footshock training. $(F)$ No effect of illumination on retention in GFP-alone control rats following the experimental parameters in $D$. 
event may be especially important (McIntyre et al. 2002; Pelletier et al. 2005). Therefore, we conducted an additional experiment in which optical inhibition was given beginning $25 \mathrm{~min}$ following the footshock training (rats were returned to their home cage during the delay), which was successful in producing higher control group latencies. Figure 3D shows the retention latencies of those rats, and a $t$-test revealed a significant difference $\left(t_{(21)}=2.781, P<0.05\right)$, indicating that inhibiting BLA axons in the VH 25 min after footshock training impaired retention. In another experiment, optical inhibition was given $3 \mathrm{~h}$ after footshock training to determine whether such inhibition produced longlasting changes in the BLA $\rightarrow$ VH pathway which could have been responsible for the impairments in retention latencies. As shown in Figure 3E, no significant difference in latencies was observed between sham-control and illumination groups $\left(t_{(31)}=1.599, \quad P>\right.$ 0.05). Finally, to determine whether the effects were due to illumination alone, another group of rats expressing GFP alone (i.e., no opsin controls) received 15 min of post-footshock training illumination at a 25-min delay. Latencies for these rats were no different from those of the sham-control group $\left(F ; t_{(13)}=\right.$ 1.252, $P>0.05)$.

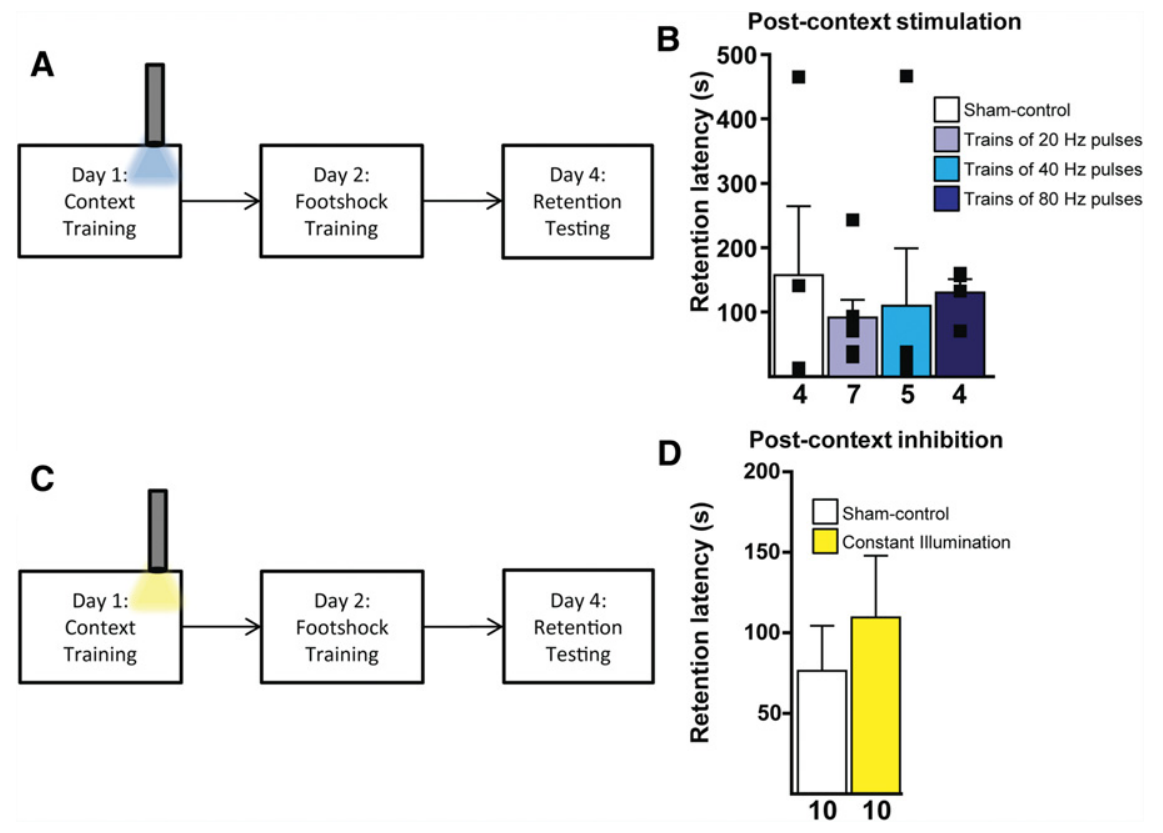

Figure 4. Retention effects of optical stimulation or inhibition given after context training on day 1 . All graphs show the mean retention latencies, in seconds ( \pm SEM), as well as the number of rats in each group under the respective bar. $(A)$ Schematic diagram of the timeline for experimental training as well as optical stimulation following context training. (B) Retention latencies of rats given post-context training optical stimulation of BLA axons in the VH via activation of ChR2(E123A). Due to the low $n$ in this experiment, the individual data points are also plotted. Such stimulation had no effect on retention regardless of stimulation frequency used. (C) Schematic diagram of the timeline for experimental training as well as optical inhibition following context training. $(D)$ Retention latencies of rats given optical inhibition in the BLA $\rightarrow$ VH pathway via activation of eArchT3.0 at a 25-min delay following context training. No effect of optical inhibition using the same parameters as Figure 3D was observed for retention.

\section{Optical stimulation and inhibition of BLA axons in the VH did not affect retention of the context learning for the modified CFC training}

Following the completion of the experiments in which optical stimulation/inhibition was given after footshock training, we conducted additional experiments to determine whether similar manipulations given after the context training (day 1) would alter retention. Rats underwent CFC training and received either post-training optical stimulation of ChR2(E123A)-transduced BLA terminals in the $\mathrm{VH}$ or post-training optical inhibition of eArchT3.0-transduced BLA terminals in the $\mathrm{VH}$ following context training on day 1 (see Fig. 4A,C for outline of training and testing). Figure $4 \mathrm{~B}$ shows retention latencies for rats that received $15 \mathrm{~min}$ of stimulation immediately following context training on day 1. A one-way ANOVA revealed no significant difference between groups regardless of stimulation frequency $\left(F_{(3,16)}=0.19\right.$, $P>0.05)$. Photoinhibition of BLA terminals in the BLA provided for $15 \mathrm{~min}$ at a 25 -min delay after context training also had no significant effect on retention (Fig. $4 \mathrm{D} ; t_{(18)}=0.70, P>0.05$ ).

Figure 5 shows histological verification of opsin expression in BLA axons in the $\mathrm{VH}$ as well as electrophysiological confirmation of robust influence of stimulation and inhibition of BLA axons (via illumination of ChR2(E123A)- and eArchT3.0-expressing BLA axons) over VH neuronal activity. Figure 5B shows representative images of the BLA (left) or BLA axons in the VH (right) expressing eArchT3.0 or ChR2(E123A), respectively. The right panel also shows a representative fiber optic track. Figure $5 \mathrm{C}$ shows a representative raster plot of neural excitation in the $\mathrm{VH}$ in response to light delivery to BLA axons in the $\mathrm{VH}$ in a ChR2(E123A)-transduced rat. Figure 5D shows a raster plot of the inhibition of BLA axons in the $\mathrm{VH}$ in a rat transduced with both ChR2(E123A) and eArchT3.0. Illumination $(473 \mathrm{~nm})$ was provided to the BLA cell bodies to stimulate BLA neurons while illumination $(561 \mathrm{~nm})$ was simultaneously provided to the $\mathrm{VH}$ to inhibit BLA axons. Inhibition was found to reduce VH neuronal activity driven by the cell body stimulation.

\section{Discussion}

The current study extends previous work examining BLA modulation of memory consolidation and provides evidence that the projection from the BLA to the $\mathrm{VH}$ plays a selective role in the consolidation of specific components of contextual fear conditioning. The findings indicate that immediate post-training optogenetic stimulation of this pathway following footshock, but not context, training increased retention latencies for CFC. This modulation was specific to stimulation using trains of 40 , but not 20 or 80, Hz light pulses. Optical inhibition of the same pathway following footshock, but not context, training impaired retention when inhibition was given $25 \mathrm{~min}$ after training. Together, these results indicate that the BLA modulates the consolidation for the footshock component of CFC through efferent projections to the $\mathrm{VH}$ and that this pathway does not play a similar role in the consolidation of the contextual learning for this task.

Prior studies indicate that the BLA modulates the consolidation for many different types of learning including inhibitory avoidance (LaLumiere et al. 2004, 2005), CFC (LaLumiere et al. 2003; Huff and Rudy 2004), conditioned taste aversion (Miranda et al. 2003; Guzman-Ramos and Bermudez-Rattoni 2012), spatial and cued-response learning (Packard et al. 1994), and novel object recognition (Roozendaal et al. 2008; Bass et al. 2012). However, 

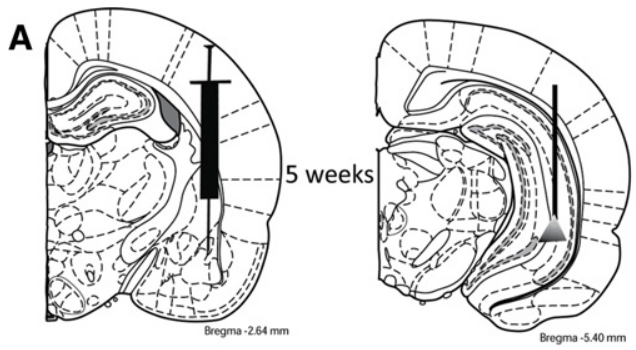

B

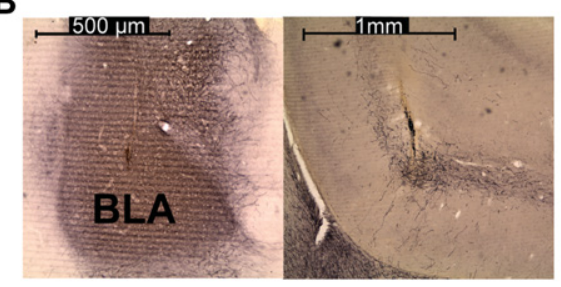

C
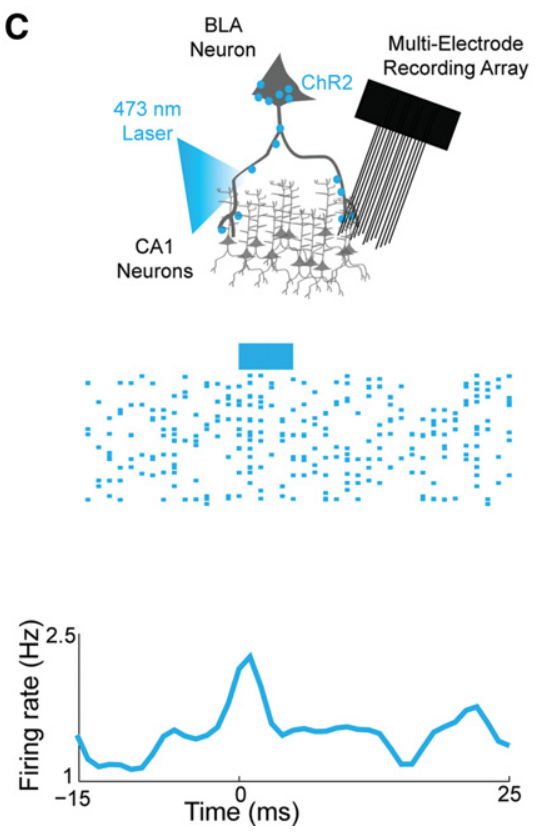

D
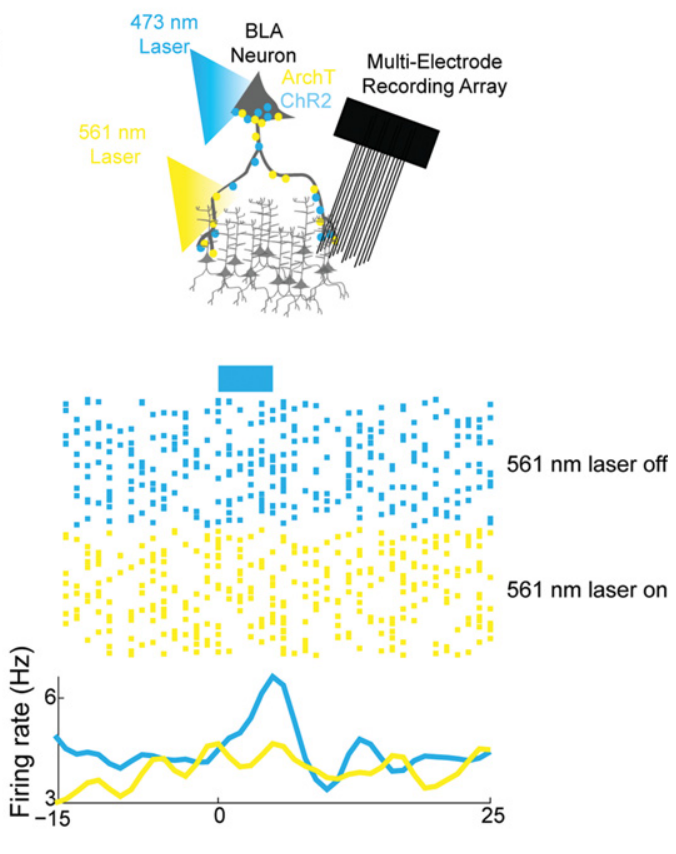

Figure 5. (A) Schematic diagram of BLA injection site, incubation time, and optic probe placement in VH. Stereotaxic images reprinted with permission from Elsevier. (B) Anti-GFP immunohistochemical staining from the injection site in a eArchT3.0-transduced rat (left) and anti-eYFP immunohistochemical staining of BLA axons in the VH in a ChR2(E123A)-transduced rat. Additionally, damage from the fiber optic probe implant can be seen terminating dorsal to the innervating fibers. (C) Raster plot of a VH neuron modulated by ChR2-transduced BLA axon stimulation. Blue rasters (above) and histogram (below) are from $40-\mathrm{Hz}$ stimulation with the 473-nm laser (ChR2-targeted). (D) Raster plot of a VH neuron modulated by both ChR2 and ArchT activation. Blue rasters (above) and histogram (below) are from 40-Hz stimulation with the 473-nm laser (ChR2-targeted). Yellow rasters (above) and histogram (below) are from simultaneous $40-\mathrm{Hz}$ stimulation with the 473-nm laser and constant illumination with the 561-nm laser (ArchT targeted).

evidence also indicates that different types or kinds of learning involve separate and distinct brain regions, as, for example, the hippocampus and caudate are involved in the consolidation of spatial and cued water maze learning, respectively (Packard et al. 1994). The BLA interacts with a variety of structures both cortical (e.g., anterior cingulate cortex, insular cortex) and subcortical (e.g., caudate, nucleus accumbens) during memory consolidation, potentially accounting for its diffuse involvement in consolidation across tasks and types of memory (for recent review, see McIntyre et al. 2012). However, prior work was unable to directly manipulate activity in distinct efferent BLA pathways to determine whether such pathways were selectively involved in specific kinds of memory consolidation.

The present work used a modified CFC paradigm, in which the consolidation of context and footshock learning are separated (Liang 1999). Prior work using this paradigm has found that, whereas the BLA itself modulates the consolidation for context and footshock components, the dorsal hippocampus and anterior cingulate are selectively involved in the consolidation for the context and footshock learning, respectively (Malin and McGaugh 2006). Thus, the present findings, indicating a role for the BLA $\rightarrow \mathrm{VH}$ pathway in the consolidation of the footshock, but not contextual, component for the task, provide evidence for the selective roles of different efferent pathways from the BLA, depending on the type of learning. Although the present work found a role for BLA projections to the VH only in footshock learning, as Malin and McGaugh observed for the anterior cingulate, there is no direct projection from the $\mathrm{VH}$ to the anterior cingulate or vice versa. Of note, however, is the consistent co-activation of the anterior cingulate and $\mathrm{VH}$ in fear memories including expression/retrieval (Cullen et al. 2015), a relationship likely mediated through the nucleus reuniens of thalamus (Vertes 2006; Nieuwenhuis and Takashima 2011). Nonetheless, a wealth of findings provide evidence supporting a promiscuous influence for the BLA on memory consolidation and more discrete roles for other brain regions depending on the component or type of learning involved. Moreover, they also suggest that the BLA, through its different projections, interacts with specific brain regions in a manner dependent on the component of the CFC investigated, a hypothesis supported by the current research.

Although the BLA is well-known for its role in modulating emotionally influenced memory consolidation, the BLA has also been shown to modulate consolidation for learning that occurs in the absence of any observable affective component, including 
object-in-context learning and contextual learning (Malin and McGaugh 2006; Barsegyan et al. 2014). However, it is impossible to rule out the possibility that any learning involves at least minimal levels of emotional arousal. Nonetheless, using the same behavioral paradigm used here, Malin and McGaugh (2006) found that post-context intra-BLA microinjections of a muscarinic cholinergic agonist enhanced retention for CFC. Thus, the present work, finding no role for the BLA $\rightarrow \mathrm{VH}$ pathway in the consolidation of the contextual learning, provides important information about the role of the BLA in memory modulation and suggests that other efferent pathways from the BLA are involved in the consolidation of the contextual learning for this CFC task.

The $\mathrm{VH}$ receives a significant innervation from the BLA and, in light of the well-established roles for the hippocampus in memory, appeared to be a likely candidate for at least some BLA influences on memory consolidation for CFC. Indeed, prior work suggests that post-training inactivation of the VH impairs CFC and auditory fear conditioning (Maren and Holt 2004). However, previous investigations have produced conflicting evidence regarding the role of the $\mathrm{VH}$ in specific aspects or types of memory (see Fanselow and Dong 2010 for larger discussion). The role of the VH in spatial information/memory processing has been unclear (Ferbinteanu and McDonald 2001; Pothuizen et al. 2004; Rudy and Matus-Amat 2005), likely due to differences in the specific regions of the hippocampus targeted or identified as "VH" as well as differences in techniques and behavioral paradigms. Yet it is generally agreed that the VH plays a significant role in emotional processing including fear and anxiety (Moser and Moser 1998; Ferbinteanu and McDonald 2001; Bannerman et al. 2003; Pothuizen et al. 2004). For example, hippocampal lesions that are confined to more ventral locations that do not connect or project to the dorsal/contextual-processing regions show reduced anxiety responses as evidenced by more time spent in the open arms of an elevated plus maze (Kjelstrup et al. 2002). Moreover, optogenetic manipulations of BLA inputs to the $\mathrm{VH}$ indicate a role for this pathway in altering anxiety-related behavior (Felix-Ortiz and Tye 2014). In agreement with some of these previous results, the present findings provide evidence that BLA inputs to the $\mathrm{VH}$ modulate the consolidation for the emotion-related aspect of contextual fear conditioning (i.e., the footshock learning) but are not involved in the contextual component. However, it is critical to note that our findings do not address the role of the $\mathrm{VH}$ itself but rather provide evidence for the role of the "pathway" from the BLA to the VH during memory consolidation.

The present findings also provide further evidence for the importance of $\gamma$ frequency activity in memory consolidation. Prior work from our laboratory indicates that optical stimulation of the BLA, using bursts of $40 \mathrm{~Hz}$ light pulses, enhances retention, whereas similar stimulation using bursts of $20-\mathrm{Hz}$ light pulses has no effect (Huff et al. 2013). The present work extends those findings to the BLA $\rightarrow \mathrm{VH}$ pathway and indicates that bursts of $80 \mathrm{~Hz}$ light pulses also have no effect. $\gamma$ Oscillations $(35-45 \mathrm{~Hz})$ are thought to be predictive of memory and underlying plasticity (Popescu et al. 2009; Headley and Weinberger 2011) and coordinated neuronal activity is suggested to play a role in regulating synaptic plasticity (Bauer et al. 2007). However, whether stimulation and inhibition of BLA axons in the $\mathrm{VH}$ is modulating underlying plasticity within the $\mathrm{VH}$ or elsewhere remains unknown. It is possible that the current modulation of BLA $\rightarrow \mathrm{VH}$ activity produces a spread of activity beyond the $\mathrm{VH}$ and that critical plasticity occurs in these downstream regions.

\section{Methodological considerations}

The target stimulation parameters chosen for these experiments were based on our previous findings indicating that the parame- ters for stimulation and inhibition are effective in modulating consolidation following a one-trial inhibitory avoidance task (Huff et al. 2013). Here, we were able to extend these stimulation parameters to the BLA $\rightarrow \mathrm{VH}$ pathway during memory consolidation of footshock learning. Importantly, a range of stimulation frequencies, including the most effective frequency $(40 \mathrm{~Hz})$ for the enhancement of consolidation for footshock learning and inhibitory avoidance learning in general (Huff et al. 2013), was used to investigate the BLA $\rightarrow \mathrm{VH}$ pathway during context learning, yet no frequency was effective for enhancing the consolidation of the context learning in this pathway. However, it remains possible that an effective frequency exists, as different frequencies of activity may be important for different types of learning. Using 4-sec trains of $20 \mathrm{~Hz}$ light pulses (to match number of light pulses in 2 -sec trains of $40 \mathrm{~Hz}$ stimulation) had no effect and implies that it is the frequency, and not the number of light pulses, that is important for the enhanced retention observed with bursts of $40 \mathrm{~Hz}$ light pulses. Nonetheless, effective parameters for other downstream targets may differ. Although a 3-h post-training control experiment was conducted for the inhibition work in order to exclude the possibility that the inhibition was producing longlasting dysfunction in the circuit, we did not perform a similar control with the stimulation experiments, as studies using pharmacological manipulations to produce memory enhancement do not observe effects on retention when given $3 \mathrm{~h}$ after training (McGaugh and Roozendaal 2009). Nonetheless, although unlikely, it is possible that the stimulation-induced memory enhancement occurred as a result of effects of the stimulation on performance during the retention test $2 \mathrm{~d}$ later, rather than on the memory consolidation.

In contrast to our previous work indicating that immediate post-training BLA inhibition impairs retention of inhibitory avoidance learning, the present study found that $15 \mathrm{~min}$ of inhibition given 25 min after training, but not immediately after training, impaired retention of the footshock learning. As we have observed, both in the present study and in our past work (Huff et al. 2013), that connecting a rat to the optical tether immediately after training reduces latencies in sham-control rats, relative to connections following a delay, this findings may reflect a "floor effect", in which latencies were too low in the control group to observe an impairment. Alternatively (and perhaps in concert with the floor effect), the present results may reflect the importance of activity in the BLA or this pathway specifically following a delay after training. Indeed, previous work suggests that emotional arousal during training leads to sustained enhancement in BLA activity for at least 30 min following stimulus presentation (Pelletier et al. 2005) and that inhibitory avoidance training induces peak norepinephrine release in the amygdala $\sim 30 \mathrm{~min}$ after training (McIntyre et al. 2002).

In contrast to many CFC studies that use freezing as the index of retention, retention in the current study was assessed via latency to cross from the illuminated compartment to the dark compartment. Because any increase in freezing would be reflected by increases in retention latencies, the use of latencies as the index of retention provides a broad measure to capture differences in memory. Nonetheless, it remains possible that our measure of retention may have failed to detect a group difference in behavior during the retention test. It is also possible that other retention measures may reflect different components of the memory that were separately modulated and yet were not observable in our particular paradigm.

\section{Conclusions}

The present study strongly indicates that the BLA projections to the VH play a dissociable role in memory consolidation, as our 
results suggest that post-training optical stimulation and inhibition of this pathway enhanced and impaired, respectively, the retention of the footshock learning. Such manipulations, however, had no effect on the retention of the context learning. These findings are, to our knowledge, the first to provide direct support for the long-held hypothesis that the modulation of memory consolidation by the BLA for so many different kinds of learning occurs through distinct efferent pathways from the BLA to downstream regions.

\section{Materials and Methods}

\section{Subjects}

Male Sprague-Dawley rats (225-250 g at time of first surgery; Charles River; $n=265$ ) were single housed in a temperaturecontrolled environment under a 12-h standard light/dark cycle (lights on at 06:00) and allowed to acclimate to the vivarium at least $3 \mathrm{~d}$ before surgery. Food and water were available ad libitum throughout all training and testing. All procedures were in compliance with NIH guidelines for care of laboratory animals and approved by the University of Iowa Institutional Animal Care and Use Committee.

\section{Surgery}

One week after arrival, rats were anesthetized using ketamine HCL $(100 \mathrm{mg} / \mathrm{kg}, \mathrm{i} . \mathrm{m}$.) and xylazine $\mathrm{HCl}(6 \mathrm{mg} / \mathrm{kg}, \mathrm{i} . \mathrm{m}$.) and placed in a stereotax (Kopf Instruments). Rats received virus microinjections [0.35 $\mu \mathrm{L}$; rAAV5-CaMKII $\alpha$-hChR2(E123A)-eYFP, the ChR2(E123A) control rAAV5-CaMKII $\alpha$-eYFP, rAAV5-CaMKII $\alpha$-eArchT3.0-GFP, or the eArchT3.0 control rAAV5-CaMKII $\alpha$-GFP; University of North Carolina Vector Core] delivered bilaterally through a 33-gauge needle into the BLA (coordinates: $2.6 \mathrm{~mm}$ posterior and $4.9 \mathrm{~mm}$ lateral to Bregma and $8.3 \mathrm{~mm}$ ventral to skull surface). The CaMKII $\alpha$ promoter limits gene expression to BLA glutamatergic cells (Tye et al. 2011). Following the virus injection, injectors were left in place for $\geq 5$ min to permit diffusion.

To allow sufficient time for opsin expression along the axons to the $\mathrm{VH}$ prior to the start of behavioral training, rats underwent a second surgery $4 \mathrm{wk}$ after the first in which fiber optic probes were bilaterally implanted, aimed at the $\mathrm{VH}$ (coordinates: $5.2 \mathrm{~mm}$ posterior and $5.5 \mathrm{~mm}$ lateral to Bregma and $7.5 \mathrm{~mm}$ ventral to skull surface) and secured by surgical screws and dental acrylic. Coordinates were based on those areas of $\mathrm{VH}$ innervated by the BLA (based on Pitkänen 2000 and modified empirically). The rats were given $1 \mathrm{wk}$ to recover from surgical procedures before behavioral training began.

\section{Optical stimulation or inhibition}

Stimulation experiments used CaMKII $\alpha$-hChR2(E123A)-eYFP and its respective control CaMKII $\alpha$-eYFP. This "ChETA" variant allows for faster off-kinetics and reliable stimulation up $200 \mathrm{~Hz}$ (Gunaydin et al. 2010; Yizhar et al. 2011). The constructs used in inhibition experiments were CaMKII $\alpha$-eArchT3.0-GFP and its respective control CaMKII $\alpha$-GFP. This modification of the outward proton pump ArchT allows for increased trafficking to the membrane, improved expression along the axon, and more rapid activation (Han et al. 2011; Mattis et al. 2012). To enable sufficient time for robust opsin expression, illumination of BLA axons in the $\mathrm{VH}$ was conducted at least 5 wk after viral injection. Figure $5 \mathrm{~A}$ shows a schematic of viral injection placement, a 5 wk incubation period, and fiber optic probe placement.

Optical probes were constructed in which the fiber optic was glued into a stainless steel ferrule assembly with one side extending out of the end of the ferrule implanted into tissue (modified from Sparta et al. 2012). The opposing side of the optical probe was polished and connected to a fiber optic leash via a ceramic split sleeve. The leash's other end (FC/PC connection) was threaded through a metal leash to protect the fiber from being damaged by the rat and attached to a 2:1 splitter allowing bilateral illumina- tion. The splitter's single end was attached to an optical commutator (Doric Lenses) allowing free rotation of the optic leashes connected to the rat. An insulated optical fiber connected the commutator to the appropriate laser source (DPSS, $300 \mathrm{~mW}, 473$ $\mathrm{nm}$ for ChR2(E123A) or $561 \mathrm{~nm}$ for eArchT3.0), with a multimode fiber coupler for an FC/PC connection. Light output was adjusted to allow for $10 \mathrm{~mW}$ at the fiber tip based on previous work (Gradinaru et al. 2009; Yizhar et al. 2011; Deisseroth 2012; Huff et al. 2013), as measured by an optical power meter. In all cases, the comparison control was a "sham-control" that had opsin expression but for which no illumination was provided except for one case (see below). Illumination was provided continuously (eArchT3.0 experiments) or was controlled by a Master-8 stimulator (ChR2(E123A) experiments; as noted in individual experiments) in a separate holding chamber following training. All stimulation and inhibition was given bilaterally.

\section{Behavioral training}

Rats were handled individually 1 min per day for $3 \mathrm{~d}$ prior to the start of training. All experiments used a standard inhibitory avoidance chamber, as depicted in Figure 1A. The apparatus was a trough-shaped box divided into two sections: one-third made of white plastic and illuminated $(30 \mathrm{~cm})$ while the second two-thirds $(60 \mathrm{~cm})$ was stainless steel and darkened. The dark chamber had a stainless steel bottom and was connected to a shock generator and timer, controlled by the experimenter. A stainless steel door, capable of being retracted through the floor, divided the two sides.

Substantial evidence from CFC studies indicates that a brief footshock with minimal time spent in a context produces poor retention of the training, an effect reversed by exposure to the context before the footshock (Fanselow 1986, 1990; Kiernan and Westbrook 1993; Landeira-Fernandez et al. 2006). Although the context and footshock are usually presented together, it is possible to present context and footshock training on separate days, allowing investigation of the separate mnemonic components of the task. Therefore, the current experiments utilized a modified CFC task, which has been described previously and successfully used to produce dissociation of context and footshock learning (Liang 1999; Malin and McGaugh 2006). Briefly, rats first underwent context training in which they were placed in the illuminated side and allowed to freely explore the entire apparatus for $3 \mathrm{~min}$ (day 1). The following day, rats were confined to the darkened side of the chamber and received an immediate inescapable footshock (day 2; time in chamber $<15 \mathrm{sec}$ ). Footshock parameters varied between stimulation (a single $1 \mathrm{~mA}, 1$-sec footshock) and inhibition (single $1 \mathrm{~mA}, 2$ sec or two $1 \mathrm{~mA}, 1$-sec footshocks) experiments to prevent ceiling and floor effects, respectively. After either context (day 1) or footshock (day 2) training, rats received optical stimulation or inhibition of BLA axons in the VH in a separate holding chamber. Specific parameters are noted in individual experiments. Retention was tested 2 d later (day 4) when rats were again placed in the illuminated compartment and allowed free access to the entire apparatus. Latency to cross into the darkened compartment (maximum $600 \mathrm{sec}$ ) was used as the index of retention.

\section{ChR2(E123A) experiments}

Immediately after either context or footshock training, rats that had been injected with the ChR2(E123A) virus received 15 min of optical stimulation of the BLA $\rightarrow \mathrm{VH}$ pathway using the following parameters: 2 -sec trains of either 20,40 , or $80-\mathrm{Hz}$ light pulses (pulse duration $=5 \mathrm{msec}$ ), given every $10 \mathrm{sec}$ as used previously (Huff et al. 2013) or, in one experiment, 4-sec trains of 20-Hz light pulses (pulse duration $=5 \mathrm{msec}$ ). For most experiments, the control group was a "sham-control" that received no illumination. However, in the main experiment, rats that had been injected with the eYFP control virus also received trains of $40-\mathrm{Hz}$ light pulses.

Based on positive results, several control experiments were conducted, most of which used only the effective stimulation parameters from the main experiment. We investigated whether the 
results with the $40-\mathrm{Hz}$ light pulses were due to the number of pulses, rather than the frequency of the pulses. Therefore, a group of rats received $20-\mathrm{Hz}$ light pulses extended to 4 -sec trains (twice the original $20-\mathrm{Hz}$ train length), every $10 \mathrm{sec}$ over $15 \mathrm{~min}$ immediately following footshock training. In a different control experiment, a group of rats received exposure to an alternate context (an operant chamber) on day 1 and 15 min of trains of $40 \mathrm{~Hz}$ pulses following footshock in the darkened side of the inhibitory avoidance chamber on day 2. Other rats that had received the ChR2(E123A) virus underwent CFC training but received no footshock during training followed by $15 \mathrm{~min}$ of trains of $40-\mathrm{Hz}$ pulses, identical to those of the main experiment.

\section{eArchT3.0 experiments}

Complementary loss-of-function experiments were conducted to investigate the "necessity" of the BLA $\rightarrow$ VH pathway for consolidation. Rats expressing eArchT3.0 received continuous optical inhibition of the BLA $\rightarrow \mathrm{VH}$ pathway for 15 min following context or footshock training, with different delays following the training. Because it was possible that the optical manipulations for the inhibition experiments were producing long-lasting dysfunction in the BLA $\rightarrow$ VH circuit that was responsible for the reduced latencies at the retention test, we conducted a control experiment in which the manipulation was given $3 \mathrm{~h}$ after training, when the consolidation window is typically observed to be closed (McGaugh and Roozendaal 2009). We did not conduct a similar control experiment with the stimulation study, as it was considered unlikely that dysfunction in the circuit created by stimulation was responsible for the observed increase in latencies at the retention test. Prior studies using pharmacological manipulations to produce memory enhancement have typically not observed effects with $3 \mathrm{~h}$ post-training manipulations (LaLumiere et al. 2004; McGaugh and Roozendaal 2009)

\section{Electrophysiological verification}

Electrophysiological activity was gathered to confirm that hippocampal neurons could be influenced by optogenetic manipulation of projections from the BLA to the $\mathrm{VH}(n=4)$. Rats were initially anesthetized with $4 \%$ isoflurane followed by i.p. injections of ketamine $(100 \mathrm{mg} / \mathrm{kg})$ and xylazine $(10 \mathrm{mg} / \mathrm{kg})$. The scalp was retracted and the skull leveled between Bregma and Lambda. Craniotomies were made at target sites in the right hemisphere. For a given rat, two sites could be optogenetically targeted to manipulate either BLA cell bodies or axons in the VH.

For verification of axon stimulation, one fiber-optic cannula was aimed at the terminal fields of BLA neurons in the $\mathrm{VH}$ $(5.1 \mathrm{~mm}$ posterior, $3.7 \mathrm{~mm}$ lateral, and $7.4 \mathrm{~mm}$ ventral to Bregma, at a $10^{\circ}$ angle in the lateral plane). For verification of inhibition, one fiber-optic cannula was lowered and fixed in place with cyanoacrylate (SloZap; Pacer Technologies) accelerated by ZipKicker (Pacer Technologies) and with methyl methacrylate (i.e., dental cement; A-M Systems). This cannula was aimed at cell bodies of BLA neurons $(2.6 \mathrm{~mm}$ posterior, $3.1 \mathrm{~mm}$ lateral, and $8.8 \mathrm{~mm}$ ventral to Bregma, at $12^{\circ}$ angle in the anterior plane) and was attached by patch cable (Doric) to a 473-nm laser (OptoEngine) driven by a pulse generator (custom-made in the Narayanan lab). The second site inhibited the terminal fields of BLA neurons in the $\mathrm{VH}(5.1 \mathrm{~mm}$ posterior, $3.7 \mathrm{~mm}$ lateral, and $7.4 \mathrm{~mm}$ ventral to Bregma, at a $10^{\circ}$ angle in the lateral plane). This cannula was attached to a patch cable (Doric) and a 561-nm laser (OptoEngine) and was held in place during the recording session by one of the two stereotactic arms. A third craniotomy was for a microwire electrode targeting layer CA1 of the $\mathrm{VH}$ (AP $-5.1, \mathrm{ML} \pm 7.1, \mathrm{DV}-7.9$ at $10^{\circ}$ in the lateral plane). Recording was done with $2 \times 8$ multielectrode arrays of $75-\mu \mathrm{m}$ tungsten wires $(250 \mu \mathrm{m}$ between wires and rows; impedance measured in vitro at $1000 \mathrm{k} \Omega$; MicroProbes for Life Science). A final craniotomy was a small hole drilled for insertion of the ground wire.

Once the electrode was lowered into location in the VH, potential neuronal units were identified on-line using an oscilloscope and audio monitor. Neuronal recordings were made using a multi-electrode recording system (Plexon). After a pause to ensure that the recording was stable, the following optogenetic protocol was initiated in order to determine whether $\mathrm{VH}$ cells responded to BLA stimulation and inhibition. Stimulation was provided $5 \mathrm{~min}$ at a time with no light, 20, or $40 \mathrm{~Hz}$ pulses of $473 \mathrm{~nm}$ light either alone or in combination with continuous $561 \mathrm{~nm}$ light.

\section{Data analysis}

Retention latencies for all behavioral experiments were analyzed using either a $t$-test or a one-way ANOVA with a Tukey post hoc test. $P<0.05$ was considered significant. All measures are expressed as mean \pm SEM, and each group's $n$ is indicated in the figure below its respective bar.

\section{Neurophysiological recordings}

Neuronal ensemble recordings in the $\mathrm{VH}$ were made using a multi-electrode recording system (Plexon). Putative single neuronal units were identified on-line using an oscilloscope and audio monitor. The Plexon off-line sorter was used to analyze waveforms off-line and to remove artifacts. Principal component analysis (PCA) and waveform shape were used for spike sorting. Single units were identified as having (1) consistent waveform shape, (2) separable clusters in PCA space, (3) average amplitude estimated at least three times larger than background activity, (4) a consistent refractory period of at least $2 \mathrm{msec}$ in interspike interval histograms, and (5) consistent firing rates around optical stimulation (as measured by a runs test of firing rates across trials around optical stimulation; neurons with $|z|$ scores $>4$ were considered "nonstationary" and were excluded). Spike activity was analyzed for all cells that fired at rates above $0.1 \mathrm{~Hz}$. Analysis of neuronal activity and quantitative analysis of basic firing properties were carried out using NeuroExplorer (Nex Technologies), and with custom routines for MATLAB.

\section{Verification of opsin expression and histology}

Rats were killed with an overdose of sodium pentobarbital $(100 \mathrm{mg} / \mathrm{ml}$; i.p.) and then perfused transcardially with phosphate-buffered saline (PBS, $\mathrm{pH}$ 7.4) followed by PBS containing $4 \%$ paraformaldehyde. Brains were removed and stored at room temperature in $4 \%$ paraformaldehyde PBS for 24-48 h until sectioning. The brains were coronally sectioned $(50 \mu \mathrm{m})$ on a vibratome and mounted onto either gelatin-subbed slides for staining or stored in anti-freeze solution at $-20^{\circ} \mathrm{C}$ until immunohistochemical procedures began. Verification of optic probes' placement was performed with a standard Nissl stain preparation (Cresyl violet) and light microscopy according to the Paxinos and Watson atlas (2005). Expression in the cell bodies and axons in the VH was verified using immunohistochemistry procedures, as described previously (Huff et al. 2013; Stefanik et al. 2013). GFP/ eYFP expression was assessed using a light microscope.

\section{Acknowledgments}

This research was supported by National Institutes of Health (NIH) grants MH097111 (R.T.L.), MH104384 (R.T.L.), and MH105187 (M.L.H.). We thank Caitlin Cosme, Andrea Gutman, Victoria Muller Ewald, Ruth Seaborne, Chelsea Ryan, and Alexandra Toft for their technical assistance. We also thank Dr. Karl Deisseroth for the Material Transfer Agreement for the opsins. The authors declare no competing financial interests.

\section{References}

Bannerman DM, Grubb M, Deacon RM, Yee BK, Feldon J, Rawlins JN. 2003. Ventral hippocampal lesions affect anxiety but not spatial learning. Behav Brain Res 139: 197-213.

Bannerman DM, Rawlins JN, McHugh SB, Deacon RM, Yee BK, Bast T, Zhang WN, Pothuizen HH, Feldon J. 2004. Regional dissociations within the hippocampus-memory and anxiety. Neurosci Biobehav Rev 28: $273-283$. 
Barrientos RM, O'Reilly RC, Rudy JW. 2002. Memory for context is impaired by injecting anisomycin into dorsal hippocampus following context exploration. Behav Brain Res 134: 299-306.

Barsegyan A, McGaugh JL, Roozendaal B. 2014. Noradrenergic activation of the basolateral amygdala modulates the consolidation of object-in-context recognition memory. Front Behav Neurosci 8: 160

Bass DI, Partain KN, Manns JR. 2012. Event-specific enhancement of memory via brief electrical stimulation to the basolateral complex of the amygdala in rats. Behav Neurosci 126: 204-208.

Bast T, Zhang WN, Feldon J. 2001. The ventral hippocampus and fear conditioning in rats. Different anterograde amnesias of fear after tetrodotoxin inactivation and infusion of the GABA(A) agonist muscimol. Exp Brain Res 139: 39-52.

Bauer EP, Paz R, Pare D. 2007. $\gamma$ Oscillations coordinate amygdalo-rhinal interactions during learning. J Neurosci 27: 9369-9379.

Chavez CM, McGaugh JL, Weinberger NM. 2013. Activation of the basolateral amygdala induces long-term enhancement of specific memory representations in the cerebral cortex. Neurobiol Learn Mem 101: $8-18$.

Cullen PK, Gilman TL, Winiecki P, Riccio DC, Jasnow AM. 2015. Activity of the anterior cingulate cortex and ventral hippocampus underlie increases in contextual fear generalization. Neurobiol Learn Mem 124: 19-27.

Deisseroth K. 2012. Predicted irradiance values: model based on direct measurements in mammalian brain tissue.

Fanselow MS. 1986. Associative vs topographical accounts of the immediate shock-freezing deficit in rats: implications for the response selection rules governing species-specific defensive reactions. Learn Motiv 17: 16-39.

Fanselow MS. 1990. Factors governing one-trial contextual conditioning. Anim Learn Behav 18: 264-270.

Fanselow MS, Dong HW. 2010. Are the dorsal and ventral hippocampus functionally distinct structures? Neuron 65: 7-19.

Felix-Ortiz AC, Tye KM. 2014. Amygdala inputs to the ventral hippocampus bidirectionally modulate social behavior. J Neurosci 34: 586-595.

Ferbinteanu J, McDonald RJ. 2001. Dorsal/ventral hippocampus, fornix, and conditioned place preference. Hippocampus 11: 187-200.

Frey S, Bergado-Rosado J, Seidenbecher T, Pape HC, Frey JU. 2001 Reinforcement of early long-term potentiation (early-LTP) in dentate gyrus by stimulation of the basolateral amygdala: heterosynaptic induction mechanisms of late-LTP. J Neurosci 21: 3697-3703.

Gilmartin MR, Kwapis JL, Helmstetter FJ. 2012. Trace and contextual fear conditioning are impaired following unilateral microinjection of muscimol in the ventral hippocampus or amygdala, but not the media prefrontal cortex. Neurobiol Learn Mem 97: 452-464.

Gradinaru V, Mogri M, Thompson KR, Henderson JM, Deisseroth K. 2009. Optical deconstruction of parkinsonian neural circuitry. Science 324: 354-359.

Gunaydin LA, Yizhar O, Berndt A, Sohal VS, Deisseroth K, Hegemann P. 2010. Ultrafast optogenetic control. Nat Neurosci 13: 387-392.

Guzman-Ramos K, Bermudez-Rattoni F. 2012. Interplay of amygdala and insular cortex during and after associative taste aversion memory formation. Rev Neurosci 23: 463-471.

Han X, Chow BY, Zhou H, Klapoetke NC, Chuong A, Rajimehr R, Yang A Baratta MV, Winkle J, Desimone R, et al. 2011. A high-light sensitivity optical neural silencer: development and application to optogenetic control of non-human primate cortex. Front Syst Neurosci 5: 18.

Headley DB, Weinberger NM. 2011. $\gamma$-Band activation predicts both associative memory and cortical plasticity. J Neurosci 31: 12748-12758.

Henke PG. 1990. Hippocampal pathway to the amygdala and stress ulcer development. Brain Res Bull 25: 691-695.

Huff NC, Rudy JW. 2004. The amygdala modulates hippocampus-dependent context memory formation and stores cue-shock associations. Behav Neurosci 118: 53-62.

Huff ML, Miller RL, Deisseroth K, Moorman DE, LaLumiere RT. 2013. Posttraining optogenetic manipulations of basolateral amygdala activity modulate consolidation of inhibitory avoidance memory in rats. Proc Natl Acad Sci 110: 3597-3602.

Ikegaya Y, Saito H, Abe K. 1995. High-frequency stimulation of the basolateral amygdala facilitates the induction of long-term potentiation in the dentate gyrus in vivo. Neurosci Res 22: 203-207.

Kiernan MJ, Westbrook RF. 1993. Effects of exposure to a to-be-shocked environment upon the rat's freezing response: evidence for facilitation, latent inhibition, and perceptual learning. Q J Exp Psychol B 46: $271-288$.

Kim JJ, Fanselow MS. 1992. Modality-specific retrograde amnesia of fear. Science 256: 675-677.

Kjelstrup KG, Tuvnes FA, Steffenach HA, Murison R, Moser EI, Moser MB. 2002. Reduced fear expression after lesions of the ventral hippocampus. Proc Natl Acad Sci 99: 10825-10830.
LaLumiere RT, Buen TV, McGaugh JL. 2003. Post-training intra-basolateral amygdala infusions of norepinephrine enhance consolidation of memory for contextual fear conditioning. J Neurosci 23: 6754-6758.

Lalumiere RT, Nguyen LT, McGaugh JL. 2004. Post-training intrabasolateral amygdala infusions of dopamine modulate consolidation of inhibitory avoidance memory: involvement of noradrenergic and cholinergic systems. Eur I Neurosci 20: 2804-2810.

LaLumiere RT, Nawar EM, McGaugh JL. 2005. Modulation of memory consolidation by the basolateral amygdala or nucleus accumbens shell requires concurrent dopamine receptor activation in both brain regions. Learn Mem 12: 296-301.

Landeira-Fernandez J, DeCola JP, Kim JJ, Fanselow MS. 2006. Immediate shock deficit in fear conditioning: effects of shock manipulations. Behav Neurosci 120: 873-879.

Liang KC. 1999. Pre- or post-training injection of buspirone impaired retention in the inhibitory avoidance task: involvement of amygdala 5-HT1A receptors. Eur J Neurosci 11: 1491-1500.

Lovitz ES, Thompson LT. 2015. Memory-enhancing intra-basolateral amygdala clenbuterol infusion reduces post-burst after hyperpolarizations in hippocampal CA1 pyramidal neurons following inhibitory avoidance learning. Neurobiol Learn Mem 119: 34-41.

Malin EL, McGaugh JL. 2006. Differential involvement of the hippocampus, anterior cingulate cortex, and basolateral amygdala in memory for context and footshock. Proc Natl Acad Sci 103: 1959-1963.

Maren S, Holt WG. 2004. Hippocampus and Pavlovian fear conditioning in rats: muscimol infusions into the ventral, but not dorsal, hippocampus impair the acquisition of conditional freezing to an auditory conditional stimulus. Behav Neurosci 118: 97-110.

Mattis J, Tye KM, Ferenczi EA, Ramakrishnan C, O'Shea DJ, Prakash R, Gunaydin LA, Hyun M, Fenno LE, Gradinaru V, et al. 2012. Principles for applying optogenetic tools derived from direct comparative analysis of microbial opsins. Nat Methods 9: 159-172.

McDonald AJ. 1991. Topographical organization of amygdaloid projections to the caudatoputamen, nucleus accumbens, and related striatal-like areas of the rat brain. Neuroscience 44: 15-33.

McGaugh JL. 2002. Memory consolidation and the amygdala: a systems perspective. Trends Neurosci 25: 456.

McGaugh JL. 2004. The amygdala modulates the consolidation of memories of emotionally arousing experiences. Annu Rev Neurosci 27: $1-28$.

McGaugh JL, Roozendaal B. 2009. Drug enhancement of memory consolidation: historical perspective and neurobiological implications. Psychopharmacology (Berl) 202: 3-14.

McIntyre CK, Hatfield T, McGaugh JL. 2002. Amygdala norepinephrine levels after training predict inhibitory avoidance retention performance in rats. Eur I Neurosci 16: 1223-1226.

McIntyre CK, Miyashita T, Setlow B, Marjon KD, Steward O, Guzowski JF McGaugh JL. 2005. Memory-influencing intra-basolateral amygdala drug infusions modulate expression of Arc protein in the hippocampus. Proc Natl Acad Sci 102: 10718-10723.

McIntyre CK, McGaugh JL, Williams CL. 2012. Interacting brain systems modulate memory consolidation. Neurosci Biobehav Rev 36: 1750-1762.

McReynolds JR, Anderson KM, Donowho KM, McIntyre CK. 2014. Noradrenergic actions in the basolateral complex of the amygdala modulate Arc expression in hippocampal synapses and consolidation of aversive and non-aversive memory. Neurobiol Learn Mem 115: 49-57.

Miranda MI, LaLumiere RT, Buen TV, Bermudez-Rattoni F, McGaugh JL. 2003. Blockade of noradrenergic receptors in the basolateral amygdala impairs taste memory. Eur J Neurosci 18: 2605-2610.

Moser MB, Moser EI. 1998. Functional differentiation in the hippocampus. Hippocampus 8: 608-619.

Moser MB, Moser EI, Forrest E, Andersen P, Morris RG. 1995. Spatial learning with a minislab in the dorsal hippocampus. Proc Natl Acad Sci 92: 9697-9701.

Nieuwenhuis IL, Takashima A. 2011. The role of the ventromedial prefrontal cortex in memory consolidation. Behav Brain Res 218: $325-334$

Packard MG, Cahill L, McGaugh JL. 1994. Amygdala modulation of hippocampal-dependent and caudate nucleus-dependent memory processes. Proc Natl Acad Sci 91: 8477-8481.

Paxinos G, Watson C. 2005. The rat brain in stereotaxic coordinates. Elsevier Academic Press, Amsterdam.

Paz R, Pelletier JG, Bauer EP, Pare D. 2006. Emotional enhancement of memory via amygdala-driven facilitation of rhinal interactions. Nat Neurosci 9: 1321-1329.

Pelletier JG, Likhtik E, Filali M, Pare D. 2005. Lasting increases in basolateral amygdala activity after emotional arousal: implications for facilitated consolidation of emotional memories. Learn Mem 12: 96-102.

Pikkarainen M, Ronkko S, Savander V, Insausti R, Pitkanen A. 1999. Projections from the lateral, basal, and accessory basal nuclei of the 
amygdala to the hippocampal formation in rat. J Comp Neurol 403: $229-260$.

Pitkänen A. 2000. Connectivity of the rat amygdaloid complex. In The amygdala (ed. Aggleton J). Oxford University Press, Oxford.

Pitkänen A, Pikkarainen M, Nurminen N, Ylinen A. 2000. Reciprocal connections between the amygdala and the hippocampal formation, perirhinal cortex, and postrhinal cortex in rat. A review. Ann N Y Acad Sci 911: 369-391.

Popescu AT, Popa D, Pare D. 2009. Coherent $\gamma$ oscillations couple the amygdala and striatum during learning. Nat Neurosci 12: 801-807.

Pothuizen HH, Zhang WN, Jongen-Relo AL, Feldon J, Yee BK. 2004. Dissociation of function between the dorsal and the ventral hippocampus in spatial learning abilities of the rat: a within-subject, within-task comparison of reference and working spatial memory. Eur $J$ Neurosci 19: 705-712.

Roozendaal B, Nguyen BT, Power AE, McGaugh JL. 1999. Basolateral amygdala noradrenergic influence enables enhancement of memory consolidation induced by hippocampal glucocorticoid receptor activation. Proc Natl Acad Sci 96: 11642-11647.

Roozendaal B, Castello NA, Vedana G, Barsegyan A, McGaugh JL. 2008. Noradrenergic activation of the basolateral amygdala modulates consolidation of object recognition memory. Neurobiol Learn Mem 90: 576-579.

Rudy JW, Matus-Amat P. 2005. The ventral hippocampus supports a memory representation of context and contextual fear conditioning: implications for a unitary function of the hippocampus. Behav Neurosci 119: $154-163$.

Sparta DR, Stamatakis AM, Phillips JL, Hovelso N, van Zessen R, Stuber GD. 2012. Construction of implantable optical fibers for long-term optogenetic manipulation of neural circuits. Nat Protoc 7: 12-23.

Stefanik MT, Moussawi K, Kupchik YM, Smith KC, Miller RL, Huff ML, Deisseroth K, Kalivas PW, LaLumiere RT. 2013. Optogenetic inhibition of cocaine seeking in rats. Addict Biol 18: 50-53.

Tye KM, Prakash R, Kim SY, Fenno LE, Grosenick L, Zarabi H, Thompson KR, Gradinaru V, Ramakrishnan C, Deisseroth K. 2011. Amygdala circuitry mediating reversible and bidirectional control of anxiety. Nature 471: 358-362.

Vertes RP. 2006. Interactions among the medial prefrontal cortex, hippocampus and midline thalamus in emotional and cognitive processing in the rat. Neuroscience 142: 1-20.

Yizhar O, Fenno LE, Davidson TJ, Mogri M, Deisseroth K. 2011. Optogenetics in neural systems. Neuron 71: 9-34.

Zhu H, Pleil KE, Urban DJ, Moy SS, Kash TL, Roth BL. 2014. Chemogenetic inactivation of ventral hippocampal glutamatergic neurons disrupts consolidation of contextual fear memory. Neuropsychopharmacology 39: $1880-1892$.

Received August 26, 2015; accepted in revised form November 18, 2015. 